\title{
ON H. WEYL'S CHARACTER FORMULA
}

\author{
BY P. CARTIER
}

Communicated by J. Dieudonné, November 22, 1960

Introduction. Many years ago, $\mathrm{H}$. Weyl [4] gave a general formula for the characters of a compact Lie group, or what amounts to the same thing, of a complex semi-simple Lie group. His proof leaned on a fundamental integration formula and was analytical and topological. Later on an algebraic proof was supplied by $H$. Freudenthal [2]. Quite recently, B. Kostant [3] gave a rather explicit formula for the multiplicity of a weight $\mu$ in an irreducible representation with maximal weight $\lambda$. The purpose of the present note is a proof for the equivalence of Weyl's and Kostant's formulae; since our proof is very simple, the benefit of Kostant's paper is a new algebraic proof for Weyl's formula. It goes without saying that [3] is of considerable independent interest for the other results it contains.

As for acknowledgments, the author is greatly indebted to the French Navy which gave him opportunity of profitable inspiration during his journey with the paratroops in Algeria.

1. Notations. Let $G$ be a complex Lie group, $\mathfrak{g}$ its Lie algebra, $B$ the Killing bilinear form on $\mathfrak{g}$, and $\mathfrak{h}$ a Cartan subalgebra of $\mathfrak{g}$. We denote by $\Sigma$ the set of roots of $\mathfrak{g}$ with respect to $\mathfrak{h}$; therefore $\Sigma$ is a set of linear forms on $\mathfrak{h}$. For each root $\alpha$ there exists a unique element $H_{\alpha}$ in $\mathfrak{h}$ such that $\alpha\left(H_{\alpha}\right)=2$ and the linear form $H \rightarrow B\left(H, H_{\alpha}\right)$ on $\mathfrak{h}$ be proportional to $\alpha$. The symmetry $S_{\alpha}$ associated to the root $\alpha$ is the linear automorphism of $\mathfrak{h}$ given by $S_{\alpha}(H)=H-\alpha(H) \cdot H_{\alpha}$; the group $W$ generated by the $S_{\alpha}$ 's is called the Weyl group; it is finite and $\Sigma$ is stable under $W$.

Let us choose now a fundamental set of roots $\Pi=\left\{\alpha_{1}, \cdots, \alpha_{l}\right\}$; that means let $\Pi$ be a set of roots and any root be a linear combination of the roots in $I I$ with integral coefficients all of the same sign; this common sign is called the sign of the root (with respect to II). By $\phi$ we mean the half-sum of all positive roots.

Let now $\pi$ be any irreducible representation of $\mathfrak{g}$ in a complex vector space $V$. For any linear form $\mu$ on $\mathfrak{h}$ let $V_{\mu}$ be the set of all $v$ in $V$ such that $\pi(H) \cdot v=\mu(H) \cdot v$ for any $H$ in $\mathfrak{h}$; then $\mu$ is a weight of $\pi$ means $V_{\mu} \neq 0$ and the multiplicity of $\mu$ is the dimension of $V_{\mu}$. As is well known, there exists a weight $\lambda$ of multiplicity 1 , such that any other weight is of the form $\lambda-\sum_{1 \leqq i \leq l} m_{i} \cdot \alpha_{i}$ with integers $m_{i} \geqq 0$ not all zero. The representation $\pi$ is defined up to equivalence by $\lambda$ and 
we write $\pi=\pi_{\lambda}$ to mean that $\lambda$ is the "maximal" weight of $\pi$. Those linear forms $\lambda$ on $\mathfrak{h}$ are candidates for a maximal weight for which $\lambda\left(H_{\alpha}\right)$ is an integer $\geqq 0$ for any $\alpha$ in $\Pi$.

2. Character formula. With the previous notations, Weyl's formula is as follows:

$$
\operatorname{Tr}\left(\pi_{\lambda}(\exp H)\right)=\frac{\sum_{s \in W} \operatorname{det} s \cdot e^{(\phi+\lambda)(s \cdot H)}}{\sum_{s \in W} \operatorname{det} s \cdot e^{\phi(s \cdot H)}} .
$$

We explain that $H$ is any element in $\mathfrak{h}$, that exp means the exponential mapping from $\mathfrak{g}$ to $G$ as defined by Chevalley [1], and $\operatorname{Tr}(A)$ is the trace of an operator $A$ on $V$. According to Weyl, the denominator in (1) can be rewritten in the following form:

$$
\sum_{s \in W} \operatorname{det} s \cdot e^{\phi(s \cdot H)}=\prod_{\alpha \text { positive root }}\left(e^{+\alpha(H) / 2}-e^{-\alpha(H) / 2}\right) .
$$

Let us now give Kostant's formula. For any linear form $\mu$ on $\mathfrak{h}$, the dimension of $V_{\mu}$ is denoted $m_{\lambda}(\mu)$ if $\lambda$ is the maximal weight of the representation $\pi$ of $\mathfrak{g}$ in $V$. Let $P(\mu)$ be the "number of partitions of $\mu$ into positive roots," that is, precisely the number of all functions $\alpha \rightarrow n_{\alpha}$ defined for positive roots $\alpha$ and with positive integral values such that $\mu=\sum_{\alpha} n_{\alpha} \cdot \alpha ; P(\mu)$ is the coefficient of $e^{-\mu}$ in the Fourier

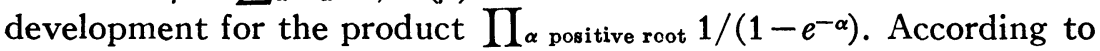
Kostant we get

$$
m_{\lambda}(\mu)=\sum_{s \in W} \operatorname{det} s \cdot P(s(\phi+\lambda)-(\phi+\mu)) .
$$

3. Proof of equivalence. For any $H$ in $\mathfrak{h}$ the operator $A=\pi_{\lambda}(\exp H)$ is diagonalizable; more precisely, on $V_{\mu}$ it induces the dilatation of ratio $e^{\mu(H)}$. Its trace is therefore equal to $\sum_{\mu} m_{\lambda}(\mu) \cdot e^{\mu(H)}$; this means $m_{\lambda}(\mu)$ is the coefficient of $e^{\mu}$ in the Fourier development for the left side of (1). Furthermore the function of $H$ given by (2) is equal to

$$
e^{\phi(H)} \cdot \prod_{\alpha \text { positive root }}\left(1-e^{-\alpha(H)}\right)
$$

and by definition of $P(\mu)$ its inverse is given by $\sum_{\nu} P(\nu) \cdot e^{-(\phi+\nu)(H)}$. For the right side of (1) we get

$$
\begin{aligned}
\sum_{\nu} & \sum_{s \in W} \operatorname{det} s \cdot e^{(\phi+\lambda)(s \cdot H)-(\phi+\nu)(\boldsymbol{H})} \cdot P(\nu) \\
& =\sum_{\mu} \sum_{s \in W} \operatorname{det} s \cdot P\left(s^{-1}(\phi+\lambda)-(\phi+\mu)\right) \cdot e^{\mu(\boldsymbol{H})}
\end{aligned}
$$


since by definition $\rho(s \cdot H)=\left(s^{-1} \rho\right)(H)$ for any $s$ in $W, H$ in $\mathfrak{h}$ and any linear form $\rho$ on $\mathfrak{h}$. Since $\operatorname{det} s= \pm 1$ for any $s$ in $W$, we get $\operatorname{det} s=\operatorname{det} s^{-1}$; therefore the right member of (3) is the coefficient of $e^{\mu}$ in the Fourier development for the right side of (1).

This finishes the proof, which looks definitely shorter than the preliminary explanations!

\section{REFERENCES}

1. C. Chevalley, Theory of Lie groups. I, Princeton University Press, 1946.

2. H. Freudenthal, Zur Berechnung der Characteren der halbeinfachen Gruppen. I, Nederl. Akad. Wetensch. Proc. Ser. A vol. 57 (1954) pp. 369-376.

3. B. Kostant, $A$ formula for the multiplicity of a weight, Trans. Amer. Math. Soc. vol. 93 (1959) pp. 53-73.

4. H. Weyl, Über die Darstellungen der halbeinfachen Gruppen durch linearen Transformationen, Math. Z. vol. 23 (1925) pp. 271-309, vol. 24 (1926) pp. 328-395.

Centre National de la Recherche Scientifique, Paris, France 